\title{
CONNECTIONS BETWEEN NOISE EQUIVALENT COUNT RATE AND IMAGE NOISE IN PET MEDICAL IMAGING
}

\author{
Vojislav Antic ${ }^{1}$, Julie Haglund ${ }^{2}$ \\ ${ }^{1}$ University Clinical Centre of Serbia, Belgrade, Serbia \\ ${ }^{2}$ Medical Physicist, Gothenburg, Sweden
}

\begin{abstract}
Assessing PET image quality is a challenge due to its clinical subjectivity and difficulties in standardisation. Methods to evaluate PET image quality include image noise and noise equivalent count rate (NECR), which are automatic and objective measurements determined from the reconstructed medical PET image or PET raw emission data from phantoms. Although manufacturers emphasize scanner performance by stating peak NECR, the peak NECR is often outside clinically relevant activity concentrations. This means that the administered activity is limited by radiation safety factors, as opposed to scanner performance. When considering image reconstruction instead, image noise in the expectation maximization (EM) algorithm is related to the true image value, so it could be inferred that this could also be true for three-dimensional ordered subset expectation maximization (3D-OSEM) algorithm. One consequence is that for the $3 D$-OSEM reconstruction, the suggested limit of injected activity, traditionally based on NECR, should be based on the true count rate. This means that images with higher SNR will be produced by increasing the injected activity to match the peak true count rate for $3 D$-OSEM [1].
\end{abstract}

Key words: PET, NECR, IN, $3 D$-OSEM, image quality, image noise

DOI: $10.21175 /$ RadProc.2016.44

\section{INTRODUCTION}

Assessing PET image quality is a challenge due to its clinical subjectivity and difficulties in standardisation. Methods to evaluate image quality in PET include image noise and noise equivalent count rate (NECR), which are automatic and objective measurements determined from the reconstructed medical PET image or PET raw emission data from phantoms.

Image noise is the standard deviation of detected pulses from a number of non-adjacent, randomly selected voxels in the region of interest. It can be reduced by increasing the total counts in the image. More counts can be obtained by increasing the scan duration, injecting more radiopharmaceutical, or improving the detection efficiency of the scanner. All of these factors are subject to some kind of limitation. Increasing the scan duration may be impractical for clinical workflow and uncomfortable for patients. Too much activity increases the radiation dose to the patient, the random coincidence counts, and dead time losses. The detection efficiency of the scanner can not be readily changed, but may necessitate using an entirely different scanner.

NECR quantifies the overall statistical usefulness of the total count rate, taking into account the effects introduced by scatter and random coincidences. NECR is widely investigated using various acquisition parameters, with the goal of obtaining the optimal value of each parameter that will yield the highest value for NECR [1].
NECR can be defined as:

$$
\mathbf{N E C R}=(\mathbf{P}-\mathbf{R})^{2} *\left(1-S^{*}\right)^{2} / \mathbf{T}+S^{\prime}+R^{\prime},
$$

where $\mathrm{P}$, prompts, is the total number of incident events, or the sum of true, scatter, and random events. $\mathrm{R}$ represents the random coincidences fraction, $\mathrm{S}^{*}$ is the scattered coincidence events, $\mathrm{T}$ is the true coincidence events, S' is the corrected frequency of scattered coincidences, and R' is the corrected frequency of random coincidences [2]. It is noted that S' and R' involve only those projections that pass through the body of the patient and are determined, respectively, from multiple random and scattered frequencies. The scattered coincidence events, $\mathrm{S}^{*}$, can be described by $\mathrm{S}^{*}=\mathrm{S} /(\mathrm{S}+\mathrm{T})$, and in this way, it is seen that $\mathrm{R}$ and $\mathrm{S}^{*}$ are derived from raw data. The true coincidence events can be expressed in terms of total, randoms, and scatter by $\mathrm{T}=(\mathrm{P}-\mathrm{R}) *\left(1-\mathrm{S}^{*}\right)[2]$.

This work addresses the connections between NECR and image noise, as well as the pitfalls of relying too much on NECR values as an indication of clinical image quality.

\section{DISCUSSION}

In PET imaging, NECR describes the true coincidence rate that would give the observed signal to noise ratio (SNR), or the same level of statistical noise, if there were no randoms and no scatter [3,4]. NECR is an estimate given an ideal system that should reach the SNR of a real system, where scatter and random coincidences are inevitable. In this way, the NECR

* antic.vojislav@gmail.com 
refers directly to the statistical quality of the projection data measurements only. NECR in general shows a linear dependence with the square root of image noise [5] and is proportional to the SNR ratio in the final reconstructed images. SNR is derived from image statistics rather than the raw data, which are needed for the NECR calculation. SNR reflects the relative signal level with respect to the noise in the reconstructed image [1].

Studies have shown that $\mathrm{SNR}^{2}$ is proportional to NECR in various situations, but the relationship is not trusted in all situations [1]. The choice of reconstruction algorithm and its associated parameters, for example, could have a large effect on image quality that could not be predicted by NECR alone. Reconstruction with OSEM may not yield a proportional relationship between $\mathrm{SNR}^{2}$ and NECR because OSEM is an iterative algorithm that provides a different noise propagation than filtered backprojection. Such noise characteristics may affect the relationship between NECR and SNR [1]. Spatial resolution and reconstruction methods frequently differ between PET systems or acquisition modes, so NECR might not always track image quality in a meaningful manner. Additionally, the raw data may not follow a Poisson distribution because of effects such as detector dead time. NECR does not account for possible count rate bias such as the systematic mispositioning of data due to spatial pile-up effects [6].

While $\mathrm{SNR}^{2}$ and NEC have been proven to be linearly related using filtered backprojection, the linear relationship may not hold true when using OSEM. The work of one group suggests that NECR might not always be predictive of SNR at high activity concentration, and a greater number of true events is not always related to better SNR [1]. Other research found a clear relationship between $\mathrm{SNR}^{2}$ and the number of true events in a phantom-based experiment with data for an OSEM-based algorithm when the NECR is not reached [1]. The correlation between $\mathrm{SNR}^{2}$ and true events was found to be higher with phantom data than with patient data, and the linear linear trend for NECR versus $\mathrm{SNR}^{2}$ was more pronounced for patients with BMI $<28 \mathrm{kgm}^{2}$ [7].

With better sinogram data, NECR increases, and for images reconstructed with the same algorithm, image quality could be expected to increase with increasing NECR. In reality, however, the quality of image regions with differing levels of counts, such as lung versus abdomen, may be affected differently [6]. In addition, each iteration of a reconstruction algorithm could uniquely and independently change the final quality of images, even for the same set of projection data [6]. NECR is therefore a limited metric with respect to assessing image quality because it is subject to what happens in the reconstruction.

With the current three-dimensional-orderedsubset-maximum-likelihood (3D-OSEM) algorithm, $\mathrm{SNR}^{2}$ versus NECR is nonlinear at activity concentrations beyond the peak NECR $[1,7]$. For the range of activity concentrations usually found in the clinic, image SNR varies with activity concentration and is dominated by the $3 \mathrm{D}-\mathrm{OSEM}$ reconstruction algorithm and associated parameters. SNR cannot be predicted by the NECR when using 3D-OSEM reconstruction, particularly for those clinical applications, such as cardiac imaging, that require high activity concentration [1]. The 3D-OSEM algorithm does not correct for scatter and random counts by subtraction of the estimated values, but rather incorporates the corrections inside the iterative loop. This approach minimizes the error propagation in correcting scatter and random counts and improves image quality.

When $\mathrm{SNR}^{2}$ versus the coincidence trues rate for the $3 \mathrm{D}-\mathrm{OSEM}$ reconstruction is plotted, the curve shows a dominant linear relationship regardless of reconstruction parameters. This suggests that scatter and randoms do not impact image quality. One important consequence is that for $3 \mathrm{D}$--OSEM reconstruction, the suggested limit of injected activity, which traditionally has been based on NECR, should actually be based on the trues count rate. It is noted that $\mathrm{SNR}^{2}$ is linearly related to the NECR before the peak NECR is reached. The NECR then decreases from its peak value at high activity concentration while the SNR squared does not track the changes of NECR, resulting in a nonlinear relationship between these two variables. This means that images with higher SNR will be produced by increasing the injected activity to match the peak trues count rate for $3 \mathrm{D}$-OSEM [1].

A linear relationship between $\mathrm{SNR}^{2}$ and NECR is not supported for $3 \mathrm{D}$-OSEM reconstruction at high activity concentration, but research hints to such a relationship between $\mathrm{SNR}^{2}$ and the trues rate. In addition, higher NECRs do not necessarily result in better image quality when $3 \mathrm{D}-\mathrm{OSEM}$ is used [1].

In order to determine an NECR-dose response model that will be capable of predicting the optimal dosage for every individual PET scan, a study was performed to correlate the administered activity and NECR [6]. The optimization criteria can be determined as a function of the peak of the NECR versus dose curve or as a fixed NECR score when NECR should be uniform across an entire patient population. The NECR-based optimization model recommends achieving $90 \%$ of peak NECR for obese patients and a considerable reduction of administered activity for normal or underweight patients so that uniform NECR is attained across the entire patient population. NECR is not proportional to injected activity after it reaches its highest peak, due to the significant increase of random events and the significant loss of trues as a result of deadtime effects at higher activities. Therefore, it has been suggested to select the minimum from all dosages corresponding to at least 90-95\% of peak NECR. The expected NECR gain beyond 90-95\% of peak is negligible, only $5-10 \%$ [6]. In comparison, there is a nearly $100 \%$ increase of dosage required to achieve that gain, so it is not recommended to introduce additional activity. This strategy would induce a large variability in NECR performance among different patient scans, since NECR is significantly affected by patient attenuation and electronics deadtime response of the scanner.

An alternative strategy is to regulate the activity in a way that the NECR is relatively constant for all patients. The maximum expected NECR for the most overweight patients could be selected as the optimization criterion. This NECR level is probably relatively low, so a prolongation of the scan duration is recommended in order to compensate for the NECR losses [6]. When comparing the NECR versus injected dose curves for patients of $70 \mathrm{~kg}$ and $38 \mathrm{~kg}$, it was 
shown that slimmer patients achieve similar NECR as obese patients, but at significantly smaller injected activities [6]. When assessing the effect of scan time on image quality, short scans using higher administered activity provided similar image quality as longer scans at lower administered activity due to the detection of more NECR statistics during the longer scan duration.

Patient-based NECR estimates can provide insight into maximum injected activities that should be used with the scanner in order to avoid operating beyond the peak NECR. Near the peak NECR, the improvement of SNR with increasing activity is very small. A very large increase in injected activity yields only a small increase in SNR when operating near the peak NECR of the scanner. This implies the possibility of a considerable reduction in injected activity without significantly compromising image quality. According to patient-based NECR estimates, for patients with large BMI, injecting more activity based on weight improves the image quality only minimally. From a view of radiation protection and image quality, it is preferred to increase the duration of the scan. NECR has been shown to fall as much as $1.4 \% / \mathrm{kg}$ with increasing patient weight [8]. For an equivalent data SNR, a 120$\mathrm{kg}$ patient would have to be scanned 2.3 times longer than a $60-\mathrm{kg}$ patient. Over this range of weight, the mean scatter fraction increases by $12 \%$ and the ratio of mean randoms to trues increases by $48 \%$ [8].

According to one study, the NECR can be reduced by a factor of 0.44 and the mean SNR by a factor of 0.66 for a $120 \mathrm{~kg}$ patient compared to a $60 \mathrm{~kg}$ patient [6]. In order to retain a similarly high NECR and image quality for both patients, it has been recommended to extend acquisition times by at least 2.3 times more for $120 \mathrm{~kg}$ patients. This avoids the alternative remedy of increasing the administered activity for overweight patients, which is beneficial because higher activities can deteriorate the NECR and image quality as well as increase the radiation exposure to patient and staff.

It must be recognized that the majority of studies and dose regimes are derived for adult patients and applied to children with the assumption that children are small adults. Due to the reduced attenuation in smaller bodies, NECR for children is expected to be very good, but the researchers Accorsi et. al. normalized and thereby sacrificed the NECR, or image quality, of pediatric patients to that of a normal, adult in order to derive dose regimes for children that reduce the injected activity and/or scan time [9]. If constant image quality is sought between adults and children, and this image quality is assessed by NRCR, then the dose to children can be reduced. Constant NECR density can be achieved with a dose or time reduction roughly proportional to weight, which corresponds to a reduction of about $50 \%$ for a patient weight of $20 \mathrm{~kg}$ [9].

Instead of optimizing injected activity on the basis of phantom measurements, clinical NECR modelling can be used. For the typical count rates found in a clinical setting, NECR and the number of true events count rate could be used as a predictive index of SNR with a nonlinear reconstruction algorithm [7]. One study used image data from 328 patients, divided into 4 BMI subgroups. In order to avoid loss of sensitivity at the edge of the field of view, the SNR was calculated within the range of 30 slices located at the center of the axial field of view that maximize the axial sensitivity.
The research revealed that the activity to be injected to achieve $90 \%$ of the NECR is almost constant, regardless of BMI. Each BMI subgroup had a unique relationship between $\mathrm{SNR}^{2}$ and NECR. A $38 \%$ increase of NECR for the BMI group 16-22 $\mathrm{kg} / \mathrm{m}^{2}$ was found to be equivalent to an increase of $93-138$ in $\mathrm{SNR}^{2}$ for this BMI group. An increase of 30\% NECR was found for the BMI group $22-28 \mathrm{~kg} / \mathrm{m}^{2}$, equivalent to an $\mathrm{SNR}^{2}$ increase of 82-119. Improvement held for the two higher BMI subgroups, although the study was limited and unconfirmed [7].

Image noise can be minimized by maximizing NECR, and manufacturers often emphasize scanner performance by stating peak NECR. Indeed, the count rate capabilities of modern PET scanners is high. In PET scanner design, there is a tradeoff between sensitivity and light decay time. Although the light output and decay constant of Lutetium based scintillators are much preferred to those characteristics of BGO, there is a small difference between the two crystal materials in stopping power and photofraction. The peak NECR is often outside clinically relevant activity concentrations. In the range of clinically used activity concentrations, the NECR of most PET scanners is not significantly different, even between BGO and L(Y)SO systems. In clinical practice, administered activity is limited by radiation safety factors instead of scanner count rate performance. PET/CT studies in normal weight patients are usually operated in the linear part of the NECR curve, where the contributions of deadtime and random coincidences have a much smaller effect on NECR than at higher FDG activities where the NECR curve becomes flat [10].

One study found similar SNR for a BGO scanner and a LYSO scanner when $3 \mathrm{D}$--OSEM reconstruction was used, even though the scanners have different NECR [2]. No statistically significant difference in the amount of image noise between the two scanners was found even though the difference in the NECR was statistically significant. This suggests that SNR may not correlate with the NECR when $3 \mathrm{D}$--OSEM reconstruction is used. When comparing BGO and LYSO scanners, a better count rate performance does not translate to a better image quality when using a 3 D-OSEM reconstruction algorithm [2].

Increasing the total number of detected net equivalent counts or increasing their detection rate when scan time is limited, as it always is in clinical practice, remain an important performance target for clinical PET systems and their associated scan protocols. A high NECR will ensure sufficient statistical quality in PET measurements for a limited acquisition period, allowing at the same time administration of radioactivity that is consistent with ALARA. The noise levels in the acquired measurements therefore constitute a non-negligible limitation for dose optimization methods [3].

\section{CONCLUSIONS}

$\mathrm{SNR}^{2}$ is in general proportional to NECR, but there are various aspects which should be taken account before indiscriminately relying on this proportionality. Factors such as scanner characteristics, acquisition mode, iterative reconstruction algorithm, injected 
activity/activity concentration, patient body type, etc. can all influence the relationship between $\mathrm{SNR}^{2}$ and NECR in different ways. Sublimation of different aspects of the relationship is crucial in order to assess the best quality of medical images, and is also significant in the development of PET technology.

Acknowledgement. This paper is a collaboration and is entirely the work of the co-authors.

\section{REFERENCES}

1. T. Chang, G. Chang, J.W. Clark Jr., R.H. Diab, E. Rohren and O.R. Mawlawi, "Reliability of Predicting Image Signal-to-Noise Ratio Using Noise Equivalent Count Rate in PET Imaging," Med. Phys., vol. 39, no. 10, pp. 5891-5900, 2012

2. T. Chang, G. Chang, S. Kohlmyer, J.W. Clark Jr., E. Rohren and O.R. Mawlawi, "Effects of Injected Dose, BMI and Scanner Type on NECR and Image Noise in PET Imaging," Phys. Med. Biol., vol. 56, no. 16, pp. 52755285, Jul. 2011

3. R.J. Ott, M.A. Flower, A.D. Hall, P.D. Marsden and J.W Babich, "Radioisotope Imaging," in, Webb's Physics of Medical Imaging, 2nd ed., M.A. Flower, Ed., Boca Raton (FL), USA: CRC Press, 2012, ch. 5, p. 335

4. E. Clementel, S. Vandenberghe, J.S. Karp and S. Surti, "Comparison of image Signal-to-Noise Ratio and Noise Equivalent Counts in Time-of-Flight PET," IEEE Nucl. Sci. Symp. Med. Imag. Conf., Knoxville (TN), USA, Oct.Nov. 2010, pp. $3622-3625,2010$
5. M. Dahlbom, C. Schiepers and J. Czernin, "Comparison of Noise Equivalent Count Rates and Image Noise," IEEE Transact. Nucl. Sci., vol. 52, no. 5, pp. 1386-1390, Oct. 2005

6. N.A. Karakatsanis, E. Fokou and C. Tsoumpas, "Dosage Optimization in Positron Emission Tomography: Stateof-the-Art Methods and Future Prospects," Am. J. Nucl. Med. Mol. Imag., vol. 5, no. 5, pp. 527-547, 2015

7. T. Carlier, L. Ferrer, H. Necib, C. Bodet-Milin, C. Rousseau and F. Kraeber-Bodéré, "Clinical NECR in 18F-FDG PET Scans: Optimization of Injected Activity and Variable Acquisition Time. Relationship with SNR," Phys. Med. Biol., vol. 59, no. 21, pp. 6417-6430, Oct. 2014

8. C.C. Watson, et al., "Optimizing Injected Dose in Clinical PET by Accurately Modeling the Counting-Rate Response Functions Specific to Individual Patient Scans," J. Nucl. Med., vol. 46, no. 11, pp. 1825-1834, Nov. 2005

9. R. Accorsi, J.S. Karp and S. Surti, "Improved Dose Regimen in Pediatric PET,” J. Nucl. Med., vol. 51, no. 2, pp. 293-300, Feb. 2010

10. E.H de Groot, N. Post, R. Boellaard, N.R.L. Wagenaar, A.T.M. Willemsen and J.A. van Dalen, "Optimized Dose Regimen for Whole-Body FDG-PET Imaging," EJNMMI Res., vol. 3, no. 63, Aug. 2013 